\title{
Möbius transform, moment-angle complexes and Halperin-Carlsson conjecture
}

\author{
Xiangyu Cao $\cdot$ Zhi Lü
}

Received: 19 October 2010 / Accepted: 19 May 2011 / Published online: 10 June 2011

(C) Springer Science+Business Media, LLC 2011

\begin{abstract}
The motivation for this paper comes from the Halperin-Carlsson conjecture for (real) moment-angle complexes. We first give an algebraic combinatorics formula for the Möbius transform of an abstract simplicial complex $K$ on $[m]=\{1, \ldots, m\}$ in terms of the Betti numbers of the Stanley-Reisner face $\operatorname{ring} \mathbf{k}(K)$ of $K$ over a field $\mathbf{k}$. We then employ a way of compressing $K$ to provide the lower bound on the sum of those Betti numbers using our formula. Next we consider a class of generalized moment-angle complexes $\mathcal{Z}_{K}^{(\mathbb{D}, \mathbb{S})}$, including the moment-angle complex $\mathcal{Z}_{K}$ and the real moment-angle complex $\mathbb{R} \mathcal{Z}_{K}$ as special examples. We show that $H^{*}\left(\mathcal{Z}_{K}^{(\mathbb{D}, \underline{S})} ; \mathbf{k}\right)$ has the same graded $\mathbf{k}$-module structure as $\operatorname{Tor}^{\mathbf{k}[\mathbf{v}]}(\mathbf{k}(K), \mathbf{k})$. Finally we show that the Halperin-Carlsson conjecture holds for $\mathcal{Z}_{K}$ (resp. $\mathbb{R} \mathcal{Z}_{K}$ ) under the restriction of the natural $T^{m}$-action on $\mathcal{Z}_{K}$ (resp. $\left(\mathbb{Z}_{2}\right)^{m}$-action on $\mathbb{R} \mathcal{Z}_{K}$ ).
\end{abstract}

Keywords Möbius transform $\cdot$ Moment-angle complex $\cdot$ Halperin-Carlsson conjecture

\section{Introduction}

Throughout this paper, assume that $m$ is a positive integer and $[m]=\{1, \ldots, m\}$. Also, $\mathbf{k}_{\ell}$ denotes the field of characteristic $\ell$ and $\mathbf{k}$ denotes a field of arbitrary char-

Supported by grants from FDUROP (No. 080705), NSFC (No. J0730103, No. 10931005) and Shanghai NSF (No. 10ZR1403600).

X. Cao

School of Mathematical Sciences, Fudan University, Shanghai, 200433, People's Republic of China e-mail: xiangyu.cao08@gmail.com

Z. Lü (凶)

School of Mathematical Sciences and The Key Laboratory of Mathematics for Nonlinear Sciences of Ministry of Education, Fudan University, Shanghai, 200433, People's Republic of China e-mail: zlu@fudan.edu.cn 
acteristic. Let

$$
2^{[m] *}=\left\{f \mid f: 2^{[m]} \longrightarrow \mathbb{Z} / 2 \mathbb{Z}=\{0,1\}\right\}
$$

consist of all $\mathbb{Z} / 2 \mathbb{Z}$-valued functions on the power set $2^{[m]}$. Then $2^{[m] *}$ forms an algebra over $\mathbb{Z} / 2 \mathbb{Z}$ in the usual way, and it has a natural basis $\left\{\delta_{a} \mid a \in 2^{[m]}\right\}$ where $\delta_{a}$ is defined as follows: $\delta_{a}(b)=1 \Longleftrightarrow b=a$. Given an element $f \in 2^{[m] *}$, the inverse image of $f$ at 1 is called the support of $f$, denoted by $\operatorname{supp}(f)$. We say that $f$ is nice if $\operatorname{supp}(f)$ is an abstract simplicial complex. Thus, we can identify all nice functions in $2^{[m] *}$ with all abstract simplicial subcomplexes in $2^{[m]}$. On $2^{[m] *}$, we then define a $\mathbb{Z} / 2 \mathbb{Z}$-valued Möbius transform $\mathcal{M}: 2^{[m] *} \longrightarrow 2^{[m] *}$ by the following way: for any $f \in 2^{[m] *}$ and $a \in 2^{[m]}, \mathcal{M}(f)(a)=\sum_{b \subseteq a} f(b)$.

Now suppose that $f \in 2^{[m] *}$ is nice such that $K=\operatorname{supp}(f)$ is an abstract simplicial complex on $[m]$. Let $\mathbf{k}(K)$ be the Stanley-Reisner face ring of $K$. The following result indicates an essential relationship between $\mathcal{M}(f)$ and the Betti numbers of $\mathbf{k}(K)$.

Theorem 1.1 (Algebraic combinatorics formula)

$$
\mathcal{M}(f)=\sum_{i=0}^{h} \sum_{a \in 2^{[m]}} \beta_{i, a}^{\mathbf{k}(K)} \delta_{a}
$$

where $h$ denotes the length of the minimal free resolution of $\mathbf{k}(K)$, and $\beta_{i, a}^{\mathbf{k}(K)}$ denote the Betti numbers of $\mathbf{k}(K)$ (see Definition 2.4).

The formula of Theorem 1.1 leads to the following inequality

$$
|\operatorname{supp}(\mathcal{M}(f))| \leq \sum_{i=0}^{h} \sum_{a \in 2^{[m]}} \beta_{i, a}^{\mathbf{k}(K)}
$$

See Corollary 3.2. Then we use an approach of compressing $\operatorname{supp}(f)$ to further analyze the lower bound on $|\operatorname{supp}(\mathcal{M}(f))|$, and the result is stated as follows.

Theorem 1.2 There exists some a $\in \operatorname{supp}(f)$ such that

$$
|\operatorname{supp}(\mathcal{M}(f))| \geq 2^{m-|a|} .
$$

Remark 1 Since $a \in \operatorname{supp}(f),|a| \leq \operatorname{dim} K+1$, so $|\operatorname{supp}(\mathcal{M}(f))| \geq 2^{m-|a|} \geq$ $2^{m-\operatorname{dim} K-1}$.

Next, associating with the Tor-algebra $\operatorname{Tor}^{\mathbf{k}}{ }^{\mathbf{v}]}(\mathbf{k}(K), \mathbf{k})$ of $\mathbf{k}(K)$, we study a class of generalized moment-angle complexes $\mathcal{Z}_{K}^{(\mathbb{D}, \mathbb{S})}$, in which the moment-angle complex $\mathcal{Z}_{K}$ and the real moment-angle complex $\mathbb{R}_{\mathcal{Z}_{K}}$ are contained, see Sect. 4.2 for the definition of $\mathcal{Z}_{K}^{(\mathbb{D}, \underline{\mathbb{S}})}$. We shall show that 
Theorem 1.3 (Theorem 4.2) $H^{*}\left(\mathcal{Z}_{K}^{(\mathbb{D}, \underline{\mathbb{S}})} ; \mathbf{k}\right)$ has the same graded $\mathbf{k}$-module structure as $\operatorname{Tor}^{\mathbf{k}[\mathbf{v}]}(\mathbf{k}(K), \mathbf{k})$.

Remark 2 Theorem 1.3 tells us that $\sum_{i} \operatorname{dim}_{\mathbf{k}} H^{i}\left(\mathcal{Z}_{K} ; \mathbf{k}\right)=\sum_{i} \operatorname{dim}_{\mathbf{k}} H^{i}\left(\mathbb{R} \mathcal{Z}_{K} ; \mathbf{k}\right)$.

As a result, we can consider the Halperin-Carlsson conjecture in the category of (real) moment-angle complexes. It is well-known that $\mathcal{Z}_{K}$ (resp. $\mathbb{R} \mathcal{Z}_{K}$ ) naturally admits a $T^{m}$-action $\Phi\left(\right.$ resp. $\left(\mathbb{Z}_{2}\right)^{m}$-action $\left.\Phi_{\mathbb{R}}\right)$.

Theorem 1.4 Let $H$ (resp. $\left.H_{\mathbb{R}}\right)$ be a rank $r$ subtorus of $T^{m}$ (resp. $\left.\left(\mathbb{Z}_{2}\right)^{m}\right)$. If the

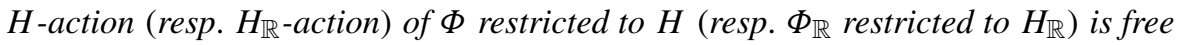
on $\mathcal{Z}_{K}$ (resp. $\left.\mathbb{R} \mathcal{Z}_{K}\right)$, then

$$
\sum_{i} \operatorname{dim}_{\mathbf{k}} H^{i}\left(\mathcal{Z}_{K} ; \mathbf{k}\right)=\sum_{i} \operatorname{dim}_{\mathbf{k}} H^{i}\left(\mathbb{R} \mathcal{Z}_{K} ; \mathbf{k}\right) \geq 2^{r} .
$$

Corollary 1.5 The Halperin-Carlsson conjecture holds for $\mathcal{Z}_{K}$ (resp. $\mathbb{R} \mathcal{Z}_{K}$ ) under the restriction of the $T^{m}$-action $\Phi\left(\right.$ resp. the $\left(\mathbb{Z}_{2}\right)^{m}$-action $\left.\Phi_{\mathbb{R}}\right){ }^{1}$

Remark 3 Following [16], the Halperin-Carlsson conjecture is stated as follows:

- Let $X$ be a finite-dimensional paracompact Hausdorff space. If $X$ admits a free action of a torus $T^{r}$ (resp. a $p$-torus $\left(\mathbb{Z}_{p}\right)^{r}, p$ prime) of rank $r$, then

$$
\sum_{i} \operatorname{dim}_{\mathbf{k}_{\ell}} H^{i}\left(X ; \mathbf{k}_{\ell}\right) \geq 2^{r}
$$

where $\ell$ is 0 (resp. $p)$.

Historically, the above conjecture in the $p$-torus case originates from the work of P.A. Smith [17]. For the case of a $p$-torus $\left(\mathbb{Z}_{p}\right)^{r}$ freely acting on a finite CWcomplex homotopic to $\left(S^{n}\right)^{k}$ this problem was suggested by P.E. Conner [10], and essential progress on it was made in $[1,7,8,18]$. In the general case, the inequality (1.1) was conjectured by S. Halperin in [13] for the torus case, and by G. Carlsson in [9] for the $p$-torus case. So far, the conjecture is known to hold $r \leq 3$ in the torus and 2-torus cases and if $r \leq 2$ in the odd $p$-torus case (see [16]). Also, many authors contributed to the conjecture in many different aspects. For more details, see, e.g., $[2,3,6,15]$.

The paper is organized as follows. In Sect. 2 we study the basic structure of the algebra $2^{[m] *}$ and the basic properties of the $\mathbb{Z} / 2 \mathbb{Z}$-valued Möbius transform, and review the notions of Stanley-Reisner face rings and their Tor-algebras. Sections 3 and 4 are two main parts of this paper. We give the proof of the algebraic combinatorics formula and estimate the lower bound on $|\operatorname{supp}(\mathcal{M}(f))|$ in Sect. 3. In Sect. 4 we review the theorem of V.M. Buchstaber and T.E. Panov on the cohomology of $\mathcal{Z}_{K}$.

\footnotetext{
${ }^{1}$ T.E. Panov informs of us that using a different method, Yury Ustinovsky has also recently proved the Halperin's toral rank conjecture for the moment-angle complexes with the restriction of natural tori actions, see arXiv:0909.1053.
} 
Then we prove Theorem 1.3 therein. Finally we finish the proof of Theorem 1.4 in Sect. 5.

\section{Möbius transform and Stanley-Reisner face ring}

\subsection{An algebra over $\mathbb{Z} / 2 \mathbb{Z}$}

Let $2^{[m]}$ denote the power set of $[m]$, which is the set of all subsets (including the empty set) of $[m]$. Then $2^{[m]}$ forms a poset with respect to inclusion $\subseteq$, and it is also a boolean algebra under the set operations of union, intersection and complement relative to $[m]$. Let

$$
2^{[m] *}=\left\{f \mid f: 2^{[m]} \longrightarrow \mathbb{Z} / 2 \mathbb{Z}=\{0,1\}\right\} .
$$

Then $2^{[m] *}$ forms an algebra over $\mathbb{Z} / 2 \mathbb{Z}$, where the addition is defined by $(f+$ $g)(a)=f(a)+g(a)$ and multiplication is defined by $(f \cdot g)(a)=f(a) g(a)$ for $a \in 2^{[m]}$. Given a function $f \in 2^{[m] *}$, define

$$
\operatorname{supp}(f):=f^{-1}(1)
$$

which is called the support of $f$.

Definition 2.1 For each $a \in 2^{[m]}$, the function $\delta_{a} \in 2^{[m] *}$ defined by

$$
\delta_{a}(b)= \begin{cases}1 & \text { if } b=a \\ 0 & \text { otherwise }\end{cases}
$$

is called the a-function. For each $i \in[m]$, the function $x_{i} \in 2^{[m] *}$ defined by

$$
x_{i}(a)=1 \quad \Leftrightarrow \quad i \in a
$$

$\forall a \in 2^{[m]}$ is called the ith coordinate function.

Lemma $2.1\left\{\delta_{a} \mid a \in 2^{[m]}\right\}$ forms a basis for $2^{[m] *}$.

Proof This is because any $f \in 2^{[m] *}$ can be expressed as

$$
f=\sum_{a \in 2^{[m]}} f(a) \delta_{a}=\sum_{a \in \operatorname{supp}(f)} \delta_{a} .
$$

By $\underline{1}$ one denotes the constant function such that $\underline{1}(a)=1$ for all $a$ in $2^{[m]}$. Obviously, $\underline{1}=\sum_{a \in 2^{[m]}} \delta_{a}$. For each $a \in 2^{[m]}$, set

$$
\mu_{a}:= \begin{cases}\prod_{i \in a} x_{i} & \text { if } a \text { is nonempty } \\ \underline{1} & \text { if } a \text { is empty. }\end{cases}
$$

Then it is easy to see that 
Lemma 2.2 Let $a, b \in 2^{[m]}$. Then $\mu_{a}(b)=1 \Leftrightarrow a \subseteq b$.

Definition 2.2 We say that $f \in 2^{[m] *}$ is nice if $\operatorname{supp}(f)$ is an abstract simplicial complex on vertex set $\bigcup_{a \in \operatorname{supp}(f)} a \subseteq[m]$. Note that an abstract simplicial complex $K$ on a subset of $[\mathrm{m}]$ is a collection of subsets in $[\mathrm{m}]$ with the property that for each $a \in K$, all subsets (including the empty set) of $a$ belong to $K$. Each $a \in K$ is called a simplex and has dimension $|a|-1$. The dimension of $K$ is defined as $\max _{a \in K}\{\operatorname{dim} a\}$.

It is easy to see that $f$ is nice if and only if for each $a \in \operatorname{supp}(f)$, any subset $b \subseteq a$ has the property $f(b)=1$.

Let $\mathcal{F}_{[m]}=\left\{f \in 2^{[m] *} \mid f\right.$ is nice $\}$, and $\mathcal{K}_{[m]}$ the set of all abstract simplicial complexes on vertex set $A$ where $A$ runs over all possible subsets in $[m]$.

Proposition 2.1 All functions of $\mathcal{F}_{[m]}$ bijectively correspond to all abstract simplicial complexes of $\mathcal{K}_{[m]}$.

Proof Clearly, $f \mapsto \operatorname{supp}(f)$ gives a bijection $\mathcal{F}_{[m]} \longrightarrow \mathcal{K}_{[m]}$, whose inverse is $K \mapsto$ $\sum_{a \in K} \delta_{a}$.

\subsection{Möbius transform}

Based upon Proposition 2.1, we shall carry out our work from the viewpoint of functional analysis.

Definition 2.3 The map $\mathcal{M}: 2^{[m] *} \longrightarrow 2^{[m] *}$ given by the formula

$$
\mathcal{M}(f)(a)=\sum_{b \subseteq a} f(b)
$$

for all $f \in 2^{[m] *}$ and $a \in 2^{[m]}$ is called the $\mathbb{Z} / 2 \mathbb{Z}$-valued Möbius transform.

Lemma 2.3 $\mathcal{M}$ is a linear transform such that $\mathcal{M}^{2}=\mathrm{id}$. In particular,

$$
\mathcal{M}\left(\delta_{a}\right)=\mu_{a}
$$

for any $a \in 2^{[m]}$. Consequently, $\mathcal{M}\left(\mu_{a}\right)=\delta_{a}$.

Proof The linearity of $\mathcal{M}$ is obvious. To check that $\mathcal{M}^{2}=\mathrm{id}$, take $f \in 2^{[\mathrm{m}] *}$, one has that for any $a \in 2^{[m]}$

$$
\mathcal{M}^{2}(f)(a)=\sum_{b \subseteq a} \sum_{c \subseteq b} f(c)=\sum_{c \subseteq a} \sum_{b \in[c, a]} f(c)=f(a)+\sum_{c \subseteq a} \sum_{b \in[c, a]} f(c) .
$$

For every term in the latter sum of (2.2), from $c \subsetneq a$ we see that $[c, a]$ is a boolean subalgebra of $2^{[m]}$ which has $2^{k}$ elements for some $k>0$. So the sum $\sum_{b \in[c, a]} f(c)=0$ in $\mathbb{Z} / 2 \mathbb{Z}$. Therefore $\mathcal{M}^{2}(f)(a)=f(a)$ for any $a \in 2^{[m]}$, so $\mathcal{M}^{2}(f)=f$ as desired. Equation (2.1) is a direct calculation by Lemma 2.2.

As a consequence of Lemmas 2.1 and 2.3, one has 
Corollary 2.1 $\left\{\mu_{a} \mid a \in 2^{[m]}\right\}$ is also a basis of $2^{[m] *}$.

Remark 4 By definition of $\mathcal{M}$, if $f(\emptyset)=1$ then $\mathcal{M}(f)(\emptyset)=1$.

In the next two subsections we shall review the Stanley-Reisner face rings and Tor-algebras. Our main reference is the book by E. Miller and B. Sturmfels [14].

\subsection{Stanley-Reisner face ring}

Now let $f \in \mathcal{F}_{[m]}$ be a nice function such that $K=\operatorname{supp}(f) \in \mathcal{K}_{[m]}$ is an abstract simplicial complex on $[m]$.

Following the notions of [14], let $\mathbf{k}[\mathbf{v}]=\mathbf{k}\left[v_{1}, \ldots, v_{m}\right]$ be the polynomial algebra over $\mathbf{k}$ on $m$ indeterminates $\mathbf{v}=v_{1}, \ldots, v_{m}$. Each monomial in $\mathbf{k}[\mathbf{v}]$ has the form of $\mathbf{v}^{\mathbf{a}}=v_{1}^{a_{1}} \cdots v_{m}^{a_{m}}$ for a vector $\mathbf{a}=\left(a_{1}, \ldots, a_{m}\right) \in \mathbb{N}^{m}$ of nonnegative integers. Thus, $\mathbf{k}[\mathbf{v}]$ is $\mathbb{N}^{m}$-graded, i.e., $\mathbf{k}[\mathbf{v}]$ is a direct sum $\bigoplus_{\mathbf{a} \in \mathbb{N}^{m}} \mathbf{k}[\mathbf{v}]_{\mathbf{a}}$ with $\mathbf{k}[\mathbf{v}]_{\mathbf{a}} \cdot \mathbf{k}[\mathbf{v}]_{\mathbf{b}}=$ $\mathbf{k}[\mathbf{v}]_{\mathbf{a}+\mathbf{b}}$ where $\mathbf{k}[\mathbf{v}]_{\mathbf{a}}=\mathbf{k}\left\{\mathbf{v}^{\mathbf{a}}\right\}$ is the vector space over $\mathbf{k}$, spanned by $\mathbf{v}^{\mathbf{a}}$. Generally, a $\mathbf{k}[\mathbf{v}]$-module $M$ is $\mathbb{N}^{m}$-graded if $M=\bigoplus_{\mathbf{b} \in \mathbb{N}^{m}} M_{\mathbf{b}}$ and $\mathbf{v}^{\mathbf{a}} \cdot M_{\mathbf{b}} \subseteq M_{\mathbf{a}+\mathbf{b}}$. Given a vector $\mathbf{a} \in \mathbb{N}^{m}$, by $\mathbf{k}[\mathbf{v}](-\mathbf{a})$ one denotes the free $\mathbf{k}[\mathbf{v}]$-module generated in degree a. So $\mathbf{k}[\mathbf{v}](-\mathbf{a})$ is isomorphic to the ideal $\left\langle\mathbf{v}^{\mathbf{a}}\right\rangle$ as $\mathbb{N}^{m}$-graded modules. Furthermore, a free $\mathbb{N}^{m}$-graded module of rank $r$ is isomorphic to the direct sum $\mathbf{k}[\mathbf{v}]\left(-\mathbf{a}_{1}\right) \oplus \cdots \oplus \mathbf{k}[\mathbf{v}]\left(-\mathbf{a}_{r}\right)$ for some vectors $\mathbf{a}_{1}, \ldots, \mathbf{a}_{r} \in \mathbb{N}^{m}$

A monomial $\mathbf{v}^{\mathbf{a}}$ in $\mathbf{k}[\mathbf{v}]$ is said to be squarefree if every coordinate of $\mathbf{a}$ is 0 or 1 , i.e., $\mathbf{a} \in\{0,1\}^{m}$ called a squarefree vector. Clearly, all elements in $2^{[m]}$ bijectively correspond to all vectors in $\{0,1\}^{m}$ by mapping $\xi: a \in 2^{[m]} \longmapsto \mathbf{a} \in\{0,1\}^{m}$, where a has entry 1 in the $i$ th place when $i \in a$, and 0 in all other entries. With this understanding, for $a \in 2^{[m]}$, one may write $\mathbf{v}^{a}=\prod_{i \in a} v_{i}$. Then the Stanley-Reisner ideal of $K$ is defined as $I_{K}=\left\langle\mathbf{v}^{\tau} \mid \tau \notin K\right\rangle$. Furthermore, the quotient ring

$$
\mathbf{k}(K)=\mathbf{k}[\mathbf{v}] / I_{K}
$$

is called the Stanley-Reisner face ring.

Example 2.1 If $K=2^{[m]}$ then $\mathbf{k}(K)=\mathbf{k}[\mathbf{v}]$, and if $K=2^{[m]} \backslash\{[m]\}$ then $\mathbf{k}(K)=$ $\mathbf{k}[\mathbf{v}] /\left\langle\mathbf{v}^{[m]}\right\rangle$.

It is well-known that $\mathbf{k}(K)$ is a finitely generated graded $\mathbf{k}[\mathbf{v}]$-module. Hilbert's syzygy theorem tells us that there exists a free resolution of $\mathbf{k}(K)$ of length at most $m$. One knows from [14, Sect. 1.4] that $\mathbf{k}(K)$ is $\mathbb{N}^{m}$-graded and it has an $\mathbb{N}^{m}$-graded minimal free resolution as follows:

$$
0 \longleftarrow \mathbf{k}(K) \longleftarrow F_{0} \stackrel{\phi_{1}}{\longleftarrow} F_{1} \longleftarrow \cdots \longleftarrow F_{h-1} \stackrel{\phi_{h}}{\longleftarrow} F_{h} \longleftarrow 0
$$

where each homomorphism $\phi_{i}$ is $\mathbb{N}^{m}$-graded degree-preserving. Since each $F_{i}$ is a free $\mathbb{N}^{m}$-graded $\mathbf{k}[\mathbf{v}]$-module, one may write $F_{i}=\bigoplus_{\mathbf{a} \in \mathbb{N}^{m}} \mathbf{k}[\mathbf{v}](-\mathbf{a})^{\beta_{i, \mathbf{a}}^{\mathbf{k}(K)}}$ where $\beta_{i, \mathbf{a}}^{\mathbf{k}(K)} \in \mathbb{N}$ (see also [14, Sect. 1.5]). By [14, Corollary 1.40], if $\mathbf{a} \in \mathbb{N}^{m}$ is not squarefree, then $\beta_{i, \mathbf{a}}^{\mathbf{k}(K)}=0$ for all $i$. Thus, we only need to consider those $\beta_{i, \mathbf{a}}^{\mathbf{k}(K)}$ with 
$\mathbf{a} \in\{0,1\}^{m}$. Throughout the following we shall write $\beta_{i, a}^{\mathbf{k}(K)}:=\beta_{i, \mathbf{a}}^{\mathbf{k}(K)}$ where $a \in 2^{[m]}$ with $\xi(a)=\mathbf{a}$.

Definition 2.4 (Cf. [14, Definition 1.29]) The number $\beta_{i, a}^{\mathbf{k}(K)}$ is called the $(i, a) t h$ Betti number of $\mathbf{k}(K)$.

\subsection{Tor-algebra of $\mathbf{k}(K)$}

Applying the functor $\otimes_{\mathbf{k}[\mathbf{v}]} \mathbf{k}$ to the sequence (2.3), one may obtain the following chain complex of $\mathbb{N}^{m}$-graded $\mathbf{k}[\mathbf{v}]$-modules:

$$
0 \longleftarrow F_{0} \otimes_{\mathbf{k}[\mathbf{v}]} \mathbf{k} \stackrel{\phi_{1}^{\prime}}{\longleftarrow} F_{1} \otimes_{\mathbf{k}[\mathbf{v}]} \mathbf{k} \longleftarrow \cdots \stackrel{\phi_{h}^{\prime}}{\longleftarrow} F_{h} \otimes_{\mathbf{k}[\mathbf{v}]} \mathbf{k} \longleftarrow 0 .
$$

Since the free resolution (2.3) is minimal, the differentials $\phi_{i}^{\prime}$ 's become zero homomorphisms. Then the $i$ th homology module of the above chain complex is $\frac{\operatorname{ker} \phi_{i}^{\prime}}{\operatorname{Im} \phi_{i+1}^{\prime}}=$ $F_{i} \otimes_{\mathbf{k}[\mathbf{v}]} \mathbf{k}$, denoted by $\operatorname{Tor}_{i}^{\mathbf{k}[\mathbf{v}]}(\mathbf{k}(K), \mathbf{k})$. Namely, $\operatorname{Tor}_{i}^{\mathbf{k}[\mathbf{v}]}(\mathbf{k}(K), \mathbf{k})=F_{i} \otimes_{\mathbf{k}[\mathbf{v}]} \mathbf{k}$ so

$$
\operatorname{dim}_{\mathbf{k}} \operatorname{Tor}_{i}^{\mathbf{k}[\mathbf{v}]}(\mathbf{k}(K), \mathbf{k})=\operatorname{rank} F_{i}=\sum_{a \in 2^{[m]}} \beta_{i, a}^{\mathbf{k}(K)}
$$

This also implies that for $\mathbf{a} \in \mathbb{N}^{m}$ with $\mathbf{a} \notin\{0,1\}^{m}, \operatorname{Tor}_{i}^{\mathbf{k}[\mathbf{v}]}(\mathbf{k}(K), \mathbf{k})_{\mathbf{a}}=0$, and so $\operatorname{Tor}_{i}^{\mathbf{k}[\mathbf{v}]}(\mathbf{k}(K), \mathbf{k})$ can be decomposed into a direct sum

$$
\bigoplus_{a \in 2^{[m]}} \operatorname{Tor}_{i}^{\mathbf{k}[\mathbf{v}]}(\mathbf{k}(K), \mathbf{k})_{a}
$$

with $\operatorname{dim}_{\mathbf{k}} \operatorname{Tor}_{i}^{\mathbf{k}[\mathbf{v}]}(\mathbf{k}(K), \mathbf{k})_{a}=\beta_{i, a}^{\mathbf{k}(K)}$ (see also [14, Lemma 1.32]). Furthermore, one has that

$$
\operatorname{Tor}^{\mathbf{k}[\mathbf{v}]}(\mathbf{k}(K), \mathbf{k})=\bigoplus_{i=0}^{h} \operatorname{Tor}_{i}^{\mathbf{k}[\mathbf{v}]}(\mathbf{k}(K), \mathbf{k})=\bigoplus_{i \in[0, h] \cap \mathbb{N}, a \in 2^{[m]}} \operatorname{Tor}_{i}^{\mathbf{k}[\mathbf{v}]}(\mathbf{k}(K), \mathbf{k})_{a}
$$

which is a bigraded $\mathbf{k}[\mathbf{v}]$-module. Combining with the above arguments, this gives

Proposition 2.2 $\sum_{i=0}^{h} \operatorname{dim}_{\mathbf{k}} \operatorname{Tor}_{i}^{\mathbf{k}[\mathbf{v}]}(\mathbf{k}(K), \mathbf{k})=\sum_{i=0}^{h} \sum_{a \in 2^{[m]}} \beta_{i, a}^{\mathbf{k}(K)}$.

\section{Möbius transform of abstract simplicial complexes and Betti numbers of face rings}

\subsection{An algebraic combinatorics formula}

Following Sects. 2.3-2.4, we now investigate the essential relationship between the Möbius transform $\mathcal{M}(f)$ of $f$ and the Betti numbers of the face ring $\mathbf{k}(K)$ of $K$. 
Theorem 3.1 (Algebraic combinatorics formula)

$$
\mathcal{M}(f)=\sum_{i=0}^{h} \sum_{a \in 2^{[m]}} \beta_{i, a}^{\mathbf{k}(K)} \delta_{a} .
$$

Proof For any $b \in 2^{[m]}$, the exact sequence (2.3) in degree $b$ reads into

$$
0 \longleftarrow \mathbf{k}^{D_{b}} \longleftarrow \mathbf{k}^{d_{b, 0}} \longleftarrow \mathbf{k}^{d_{b, 1}} \longleftarrow \mathbf{k}^{d_{b, 2}} \longleftarrow \cdots \longleftarrow \mathbf{k}^{d_{b, h}} \longleftarrow 0
$$

where $D_{b}=\operatorname{dim}_{\mathbf{k}} \mathbf{k}(K)_{b}$ and $d_{b, i}=\operatorname{dim}_{\mathbf{k}}\left(F_{i}\right)_{b}$. Since the above sequence is also exact, we have $D_{b}=\sum_{i=0}^{h}(-1)^{i} d_{i, b}$. An easy observation shows that $f(b)=$ $\operatorname{dim}_{\mathbf{k}} \mathbf{k}(K)_{b}=D_{b}$, and $d_{b, i}=\sum_{a \subseteq b} \beta_{i, a}^{\mathbf{k}(K)}$ (this is induced from $F_{i}=$ $\left.\bigoplus_{\mathbf{a} \in \mathbb{N}^{m}} \mathbf{k}[\mathbf{v}](-\mathbf{a})^{\beta_{i, \mathbf{a}}^{\mathbf{k}(K)}}\right)$.

Now let us work in integers modulo 2 . We then have $D_{b}=\sum_{i=0}^{h} d_{i, b}$, and further

$$
f(b)=\sum_{i=0}^{h} \sum_{a \subseteq b} \beta_{i, a}^{\mathbf{k}(K)}=\sum_{i=0}^{h} \sum_{a \in 2^{[m]}} \beta_{i, a}^{\mathbf{k}(K)} \mu_{a}(b) .
$$

So

$$
f=\sum_{i=0}^{h} \sum_{a \in 2^{[m]}} \beta_{i, a}^{\mathbf{k}(K)} \mu_{a}
$$

Applying $\mathcal{M}$ to the above equality and noting that $\mathcal{M}\left(\mu_{a}\right)=\delta_{a}$, we arrive at the required formula.

Corollary 3.2 Let $f \in 2^{[m] *}$ be a nice function such that $K=\operatorname{supp}(f) \in \mathcal{K}_{[m]}$ is an abstract simplicial complex on $[m]$. Then

$$
|\operatorname{supp}(\mathcal{M}(f))| \leq \sum_{i=0}^{h} \sum_{a \in 2^{[m]}} \beta_{i, a}^{\mathbf{k}(K)} .
$$

Proof From the formula of Theorem 3.1, one has

$$
\mathcal{M}(f)=\sum_{i=0}^{h} \sum_{a \in 2^{[m]}} \beta_{i, a}^{\mathbf{k}(K)} \delta_{a}=\sum_{a \in 2^{[m]}}\left(\sum_{i=0}^{h} \beta_{i, a}^{\mathbf{k}(K)}\right) \delta_{a}
$$

so for any $a \in \operatorname{supp}(\mathcal{M}(f)), \sum_{i=0}^{h} \beta_{i, a}^{\mathbf{k}(K)}$ must be odd and nonnegative, and then $\sum_{i=0}^{h} \beta_{i, a}^{\mathbf{k}(K)} \geq 1$. Therefore

$$
\sum_{i=0}^{h} \sum_{a \in 2^{[m]}} \beta_{i, a}^{\mathbf{k}(K)} \geq \sum_{a \in \operatorname{supp}(\mathcal{M}(f))} \sum_{i=0}^{h} \beta_{i, a}^{\mathbf{k}(K)} \geq \sum_{a \in \operatorname{supp}(\mathcal{M}(f))} 1=|\operatorname{supp}(\mathcal{M}(f))|
$$

as desired. 
3.2 The estimation of the lower bound on $|\operatorname{supp}(\mathcal{M}(f))|$

We shall upbuild a method of compressing $\operatorname{supp}(f)$ to get the desired lower bound on $|\operatorname{supp}(\mathcal{M}(f))|$.

Definition 3.1 Fix $k \in[m]$. We say that $f \in \mathcal{F}_{[m]}$ is $k$ th extendable if

(k-1) $f(\{k\})=1$;

(k-2) $\mathcal{M}(f) \cdot x_{k} \neq 0$ in $2^{[m] *}$.

The linear transformation $E_{k}: 2^{[m] *} \longrightarrow 2^{[m] *}$ determined by $\mu_{a} \mapsto \mu_{a \backslash\{k\}}$ is called the kth compression-operator. A function $f \in \mathcal{F}_{[m]}$ is said to be extendable if it is $k$ th extendable for some $k \in[m]$; otherwise, $f$ is said to be non-extendable.

Introducing the map $\epsilon_{k}: 2^{[m]} \longrightarrow 2^{[m]}$ defined by $a \mapsto a \cup\{k\}$, we derive the following formula for $E_{k}$.

Lemma 3.1 For any $f \in 2^{[m] *}$ we have

$$
E_{k}(f)=f \circ \epsilon_{k}
$$

Proof It suffices to check that the formula $E_{k}\left(\mu_{a}\right)=\mu_{a} \circ \epsilon_{k}$ holds for each $a \in 2^{[m]}$. Indeed, take $b \in 2^{[m]}$, we have

$$
E_{k}\left(\mu_{a}\right)(b)=1 \quad \Leftrightarrow \quad a \backslash\{k\} \subseteq b \quad \Leftrightarrow \quad a \subseteq b \cup\{k\} \quad \Leftrightarrow \quad \mu_{a}\left(\epsilon_{k}(b)\right)=1 .
$$

Therefore, $E_{k}\left(\mu_{a}\right)=\mu_{a} \circ \epsilon_{k}$ as desired.

Remark 5 We see from Lemma 3.1 that $E_{k}$ is exactly the star operator at $k$.

Proposition 3.1 Fix $k \in[m]$. If $f \in \mathcal{F}_{[m]}$ satisfies $f(\{k\})=1$, then $E_{k}(f) \in \mathcal{F}_{[m]}$ and $\operatorname{supp}\left(E_{k}(f)\right) \subseteq \operatorname{supp}(f)$.

Proof For any pair $a \subseteq b$ in $2^{[m]}$, we have $\epsilon_{k}(a) \subseteq \epsilon_{k}(b)$. So if $E_{k}(f)(b)=$ $f\left(\epsilon_{k}(b)\right)=1$, then $f\left(\epsilon_{k}(a)\right)=1$ since $f \in \mathcal{F}_{[m]}$, and so $E_{k}(f)(a)=1$. Also, $f(\{k\})=1$ implies that $E_{k}(f)(\emptyset)=f(\emptyset \cup\{k\})=1$. Thus, $E_{k}(f)$ is nice.

For any $a \in 2^{[m]}$, if $E_{k}(f)(a)=1$ then by Lemma 3.1 $f\left(\epsilon_{k}(a)\right)=1$, so $f(a)=1$ since $a \subseteq \epsilon_{k}(a)$ and $f \in \mathcal{F}_{[m]}$. Hence, $\operatorname{supp}\left(E_{k}(f)\right) \subseteq \operatorname{supp}(f)$ as desired.

Now let us look at the composition transformation $\mathcal{M} \circ E_{k} \circ \mathcal{M}=: \hat{E}_{k}$. For any $a \in 2^{[m]}$, one has

$$
\hat{E}_{k}\left(\delta_{a}\right)=\mathcal{M} \circ E_{k} \circ \mathcal{M}\left(\delta_{a}\right)=\mathcal{M} \circ E_{k}\left(\mu_{a}\right)=\mathcal{M}\left(\mu_{a \backslash\{k\}}\right)=\delta_{a \backslash\{k\}} .
$$

Note also that since $\mathcal{M}^{2}=\mathrm{id}, \mathcal{M} \circ E_{k}=\hat{E}_{k} \circ \mathcal{M}$.

Lemma 3.2 For any $g \in 2^{[m] *}$ and $k \in[m]$, we have $\hat{E}_{k}(g) x_{k}=0$. 
Proof Write $g=\sum_{a \in \operatorname{supp}(g)} \delta_{a}$. Since $\hat{E}_{k}$ is linear and $\hat{E}_{k}\left(\delta_{a}\right)=\delta_{a \backslash\{k\}}$ for any $a \in 2^{[m]}$, it follows that $\hat{E}_{k}(g)=\sum_{a \in \operatorname{supp}(g)} \delta_{a \backslash\{k\}}$. Obviously, for any $a \in 2^{[m]}$, $\delta_{a \backslash\{k\}} x_{k}=0$. Thus, $\hat{E}_{k}(g) x_{k}=0$ as desired.

Corollary 3.3 Let $k \in[m]$. If $f \in 2^{[m] *}$ satisfies $\mathcal{M}(f) x_{k} \neq 0$, then $f \neq E_{k}(f)$.

Proof Suppose that $f=E_{k}(f)$. Applying $\mathcal{M}$ to both sides, we get $\mathcal{M}(f)=$ $\mathcal{M}\left(E_{k}(f)\right)=\hat{E}_{k}(\mathcal{M}(f))$. Write $g=\mathcal{M}(f)$. Then $g=\hat{E}_{k}(g)$. Multiplying by $x_{k}$ on the two sides of $g=\hat{E}_{k}(g)$, we have $g x_{k}=\hat{E}_{k}(g) x_{k}$. Since $g x_{k}=\mathcal{M}(f) x_{k} \neq 0$, we have $\hat{E}_{k}(g) x_{k} \neq 0$, a contradiction by Lemma 3.2 .

Proposition 3.2 Let $f \in 2^{[m] *}$. Then for each $k \in[m]$,

$$
\left|\operatorname{supp}\left(\hat{E}_{k}(f)\right)\right| \leq|\operatorname{supp}(f)| \text {. }
$$

Proof Let $A=\left\{a \in 2^{[m]} \mid k \notin a, a \in \operatorname{supp}(f)\right\}$ and $B=\left\{a \in 2^{[m]} \mid k \notin a, \epsilon_{k}(a) \in\right.$ $\operatorname{supp}(f)\}$. Then we have

$$
f=\sum_{a \in \operatorname{supp}(f)} \delta_{a}=\sum_{\substack{a \in \operatorname{supp}(f) \\ k \notin a}} \delta_{a}+\sum_{\substack{a \in \operatorname{supp}(f) \\ k \in a}} \delta_{a}=\sum_{a \in A} \delta_{a}+\sum_{a \in B} \delta_{\epsilon_{k}(a)}
$$

and by (3.1)

$$
\hat{E}_{k}(f)=\sum_{a \in A} \delta_{a \backslash\{k\}}+\sum_{a \in B} \delta_{\epsilon_{k}(a) \backslash\{k\}}=\sum_{a \in A} \delta_{a}+\sum_{a \in B} \delta_{a}=\sum_{a \in A \triangle B} \delta_{a}
$$

where $A \triangle B=(A \backslash B) \cup(B \backslash A)$. Now

$$
\left|\operatorname{supp}\left(\hat{E}_{k}(f)\right)\right|=|A \triangle B| \leq|A|+|B|=|\operatorname{supp}(f)|
$$

as desired.

Remark 6 Observe that for any $f \in \mathcal{F}_{[m]}$, whenever $f$ is $k$ th extendable for some $k \in[m]$, by Proposition 3.1 and Corollary 3.3 we obtain that $E_{k}(f) \in \mathcal{F}_{[m]}$ and $\operatorname{supp}\left(E_{k}(f)\right) \subsetneq \operatorname{supp}(f)$. In addition, since $\left(\mathcal{M} \circ E_{k}\right)(f)=\left(\hat{E}_{k} \circ \mathcal{M}\right)(f)$, by Proposition 3.2 one has that $\left|\operatorname{supp}\left(\mathcal{M}\left(E_{k}(f)\right)\right)\right| \leq|\operatorname{supp}(\mathcal{M}(f))|$. We replace $f$ with $E_{k}(f)$ and repeat the above process whenever possible, so as to get a sequence of functions in $\mathcal{F}_{[m]}$ with strictly decreasing support. This process must end after a finite number of steps, giving finally a $f_{0} \in \mathcal{F}_{[m]}$ that is non-extendable with $\operatorname{supp}\left(f_{0}\right) \subseteq \operatorname{supp}(f)$ and $\left|\operatorname{supp}\left(\mathcal{M}\left(f_{0}\right)\right)\right| \leq|\operatorname{supp}(\mathcal{M}(f))|$. It remains to characterize such a non-extendable $f_{0} \in \mathcal{F}_{[m]}$.

Proposition 3.3 Let $f \in \mathcal{F}_{[m]}$. Then $f$ is non-extendable if and only if there is some $a_{0} \in 2^{[m]}$ such that $\operatorname{supp}(f)=2^{a_{0}}$ (i.e., $f=\sum_{b \subseteq a_{0}} \delta_{b}$ ).

Proof Suppose that $f$ is non-extendable. Let $a_{0}=\{k \in[m] \mid f(\{k\})=1\}$. If $a_{0}=\emptyset$, obviously we have $f=\delta_{\emptyset}$. Assume that $a_{0}$ is non-empty. Given an element $b \in 2^{[\mathrm{m}]}$, 
if $f(b)=1$, since $f \in \mathcal{F}_{[m]}$, then for any $k \in b, f(\{k\})=1$ so $k \in a_{0}$ and $b \subseteq a_{0}$. Since $f$ is non-extendable, $\mathcal{M}(f) x_{k}=0$ for any $k \in a_{0}$. Then we see from $\mathcal{M}(f)=$ $\sum_{b \in \operatorname{supp}(\mathcal{M}(f))} \delta_{b}$ that for any $b \in \operatorname{supp}(\mathcal{M}(f)), b \cap a_{0}=\emptyset$. Since $\mathcal{M}(f)(\emptyset)=1$, we have $\emptyset \in \operatorname{supp}(\mathcal{M}(f))$. Furthermore

$$
\begin{aligned}
f\left(a_{0}\right) & =\mathcal{M}^{2}(f)\left(a_{0}\right)=\mathcal{M}\left(\sum_{b \in \operatorname{supp}(\mathcal{M}(f))} \delta_{b}\right)\left(a_{0}\right) \\
& =\sum_{b \in \operatorname{supp}(\mathcal{M}(f))} \mu_{b}\left(a_{0}\right)=\mu_{\emptyset}\left(a_{0}\right)=1 .
\end{aligned}
$$

Since $f \in \mathcal{F}_{[m]}$, it follows that for any subset $b \subseteq a_{0}, f(b)=1$. Therefore, for $b \in$ $2^{[m]}$

$$
f(b)=1 \quad \Leftrightarrow \quad b \subseteq a_{0} .
$$

This implies that $\operatorname{supp}(f)=2^{a_{0}}=\left\{b \in 2^{[m]} \mid b \subseteq a_{0}\right\}$.

Conversely, suppose that $f=\sum_{b \subseteq a_{0}} \delta_{b}$ for some $a_{0} \in[m]$. If $a_{0}=[m]$, then $f=\underline{1}=\mu_{\emptyset}$ so $\mathcal{M}(f)=\delta_{\emptyset}$. Moreover, for any $k \in a_{0}, \mathcal{M}(f) x_{k}=0$ so $f$ is nonextendable. If $a_{0}=\emptyset$, obviously $f$ is non-extendable. Assume that $a_{0} \neq[\mathrm{m}], \emptyset$. Then an easy argument shows that

$$
f=\prod_{i \in[m] \backslash a_{0}}\left(\underline{1}+x_{i}\right)=\sum_{b \subseteq[m] \backslash a_{0}} \mu_{b} .
$$

Applying $\mathcal{M}$ to the above equality, it follows that $\mathcal{M}(f)=\sum_{b \subseteq[m] \backslash a_{0}} \delta_{b}$. Now for any $k \in a_{0}$ and any $b \subseteq[m] \backslash a_{0}$, we have $\delta_{b} x_{k}=0$ so $\mathcal{M}(f) x_{k}=0$. This means that $f$ is also non-extendable.

From the proof of Proposition 3.3, we easily see that

Corollary 3.4 Let $a \in 2^{[m]}$. Then $f=\sum_{b \subseteq a} \delta_{b}$ if and only if $\mathcal{M}(f)=\sum_{b \subseteq[m] \backslash a} \delta_{b}$ (i.e., $\operatorname{supp}(f)=2^{a}$ if and only if $\left.\operatorname{supp}(\mathcal{M}(f))=2^{[m] \backslash a}\right)$. In this case, $|\operatorname{supp}(\mathcal{M}(f))|=2^{m-|a|}$.

We now summarize the above arguments as follows.

Theorem 3.5 For any $f \in \mathcal{F}_{[m]}$, there exists some $a \in \operatorname{supp}(f)$ such that

$$
|\operatorname{supp}(\mathcal{M}(f))| \geq 2^{m-|a|} .
$$

Remark 7 The interested readers are invited to see a simple fact that $f \in \mathcal{F}_{[m]}$ can be compressed by compression-operators into a non-extendable $f_{0}$ with $\operatorname{supp}\left(f_{0}\right)=2^{a_{0}}$ if and only if $a_{0}$ is a maximal element in $\operatorname{supp}(f)$ as a poset. This result will not be used later in this article. 


\section{Moment-angle complexes and their cohomologies}

Let $K$ be an abstract simplicial complex on vertex set $[m]$. Let $(X, W)$ be a pair of topological spaces with $W \subset X$. Following [5, Construction 6.38], for each simplex $\sigma$ in $K$, set

$$
B_{\sigma}(X, W)=\prod_{i=1}^{m} A_{i}
$$

such that

$$
A_{i}= \begin{cases}X & \text { if } i \in \sigma, \\ W & \text { if } i \in[m] \backslash \sigma .\end{cases}
$$

Then one can define the following subspace of the product space $X^{m}$ :

$$
K(X, W)=\bigcup_{\sigma \in K} B_{\sigma}(X, W) \subset X^{m} .
$$

4.1 Moment-angle complexes

When the pair $(X, W)$ is chosen as $\left(D^{2}, S^{1}\right)$,

$$
\mathcal{Z}_{K}:=K\left(D^{2}, S^{1}\right) \subset\left(D^{2}\right)^{m}
$$

is called the moment-angle complex on $K$ where $D^{2}=\{z \in \mathbb{C}|| z \mid \leq 1\}$ is the unit disk in $\mathbb{C}$, and $S^{1}=\partial D^{2}$. Since $\left(D^{2}\right)^{m} \subset \mathbb{C}^{m}$ is invariant under the standard action of $T^{m}$ on $\mathbb{C}^{m}$ given by

$$
\left(\left(g_{1}, \ldots, g_{m}\right),\left(z_{1}, \ldots, z_{m}\right)\right) \longmapsto\left(g_{1} z_{1}, \ldots, g_{m} z_{m}\right),
$$

$\left(D^{2}\right)^{m}$ admits a natural $T^{m}$-action whose orbit space is the unit cube $I^{m} \subset \mathbb{R}_{\geq 0}^{m}$. The action $T^{m} \curvearrowright\left(D^{2}\right)^{m}$ then induces a canonical $T^{m}$-action $\Phi$ on $\mathcal{Z}_{K}$.

When the pair $(X, W)$ is chosen as $\left(D^{1}, S^{0}\right)$,

$$
\mathbb{R} \mathcal{Z}_{K}:=K\left(D^{1}, S^{0}\right) \subset\left(D^{1}\right)^{m}
$$

is called the real moment-angle complex on $K$ where $D^{1}=\{x \in \mathbb{R}|| x \mid \leq 1\}=[-1,1]$ is the unit disk in $\mathbb{R}$, and $S^{0}=\partial D^{1}=\{ \pm 1\}$. Similarly, $\left(D^{1}\right)^{m} \subset \mathbb{R}^{m}$ is invariant under the standard action of $\left(\mathbb{Z}_{2}\right)^{m}$ on $\mathbb{R}^{m}$ given by

$$
\left(\left(g_{1}, \ldots, g_{m}\right),\left(x_{1}, \ldots, x_{m}\right)\right) \longmapsto\left(g_{1} x_{1}, \ldots, g_{m} x_{m}\right) .
$$

Thus $\left(D^{1}\right)^{m}$ admits a natural $\left(\mathbb{Z}_{2}\right)^{m}$-action whose orbit space is also the unit cube $I^{m} \subset \mathbb{R}_{\geq 0}^{m}$, where $\mathbb{Z}_{2}=\{-1,1\}$ is the group with respect to multiplication. Furthermore, the action $\left(\mathbb{Z}_{2}\right)^{m} \curvearrowright\left(D^{1}\right)^{m}$ also induces a canonical $\left(\mathbb{Z}_{2}\right)^{m}$-action $\Phi_{\mathbb{R}}$ on $\mathbb{R} \mathcal{Z}_{K}$.

Let $P_{K}$ be the cone on the barycentric subdivision of $K$. Since the cone on the barycentric subdivision of a $k$-simplex is combinatorially equivalent to the standard subdivision of a $(k+1)$-cube, $P_{K}$ is naturally a cubical complex and it is decomposed into cubes indexed by the simplices of $K$. Then one knows from [5] and [11] that both $T^{m}$-action $\Phi$ on $\mathcal{Z}_{K}$ and $\left(\mathbb{Z}_{2}\right)^{m}$-action $\Phi_{\mathbb{R}}$ on $\mathbb{R} \mathcal{Z}_{K}$ have the same orbit space $P_{K}$. 
Example 4.1 When $K=2^{[m]}, \mathcal{Z}_{K}=\left(D^{2}\right)^{m}$ and $\mathbb{R} \mathcal{Z}_{K}=\left(D^{1}\right)^{m}$. When $K=2^{[m]} \backslash$ $\{[m]\}, \mathcal{Z}_{K}=S^{2 m-1}$ and $\mathbb{R} \mathcal{Z}_{K}=S^{m-1}$.

Remark 8 In general, $\mathcal{Z}_{K}$ and $\mathbb{R} \mathcal{Z}_{K}$ are not manifolds. However, if $K$ is a simplicial sphere, then both $\mathcal{Z}_{K}$ and $\mathbb{R} \mathcal{Z}_{K}$ are closed manifolds (see [5, Lemma 6.13]).

\subsection{Cohomology}

V.M. Buchstaber and T.E. Panov in [5, Theorem 7.6] have calculated the cohomology of $\mathcal{Z}_{K}$ (see also [15, Theorem 4.7]). Their result is stated as follows.

Theorem 4.1 (Buchstaber-Panov) As k-algebras,

$$
H^{*}\left(\mathcal{Z}_{K} ; \mathbf{k}\right) \cong \operatorname{Tor}^{\mathbf{k}[\mathbf{v}]}(\mathbf{k}(K), \mathbf{k})
$$

where $\mathbf{k}(K)=\mathbf{k}[\mathbf{v}] / I_{K}=\mathbf{k}\left[v_{1}, \ldots, v_{m}\right] / I_{K}$ with $\operatorname{deg} v_{i}=2$.

Here we calculate the cohomologies of a class of generalized moment-angle complexes. For this, we begin with the notion of the generalized moment-angle complex, due to N. Strickland, cf. [4] and [12]. Given an abstract simplicial complex $K$ on $[m]$, let $(\underline{X}, \underline{W})=\left\{\left(X_{i}, W_{i}\right)\right\}_{i=1}^{m}$ be $m$ pairs of CW-complexes with $W_{i} \subset X_{i}$. Then the generalized moment-angle complex is defined as follows:

$$
K(\underline{X}, \underline{W})=\bigcup_{\sigma \in K} B_{\sigma}(\underline{X}, \underline{W}) \subset \prod_{i=1}^{m} X_{i}
$$

where $B_{\sigma}(\underline{X}, \underline{W})=\prod_{i=1}^{m} H_{i}$ and

$$
H_{i}= \begin{cases}X_{i} & \text { if } i \in \sigma \\ W_{i} & \text { if } i \in[m] \backslash \sigma .\end{cases}
$$

Now take $(\underline{X}, \underline{W})=(\underline{\mathbb{D}}, \underline{\mathbb{S}})=\left\{\left(\mathbb{D}_{i}, \mathbb{S}_{i}\right)\right\}_{i=1}^{m}$ with each CW-complex pair $\left(\mathbb{D}_{i}, \mathbb{S}_{i}\right)$ subject to the following conditions:

(1) $\mathbb{D}_{i}$ is acyclic, that is, $\widetilde{H}_{j}\left(\mathbb{D}_{i}\right)=0$ for any $j$.

(2) There exists a unique $\kappa_{i}$ such that $\widetilde{H}_{\kappa_{i}}\left(\mathbb{S}_{i}\right)=\mathbb{Z}$ and $\widetilde{H}_{j}\left(\mathbb{S}_{i}\right)=0$ for any $j \neq \kappa_{i}$.

Then our objective is to calculate the cohomology of

$$
\mathcal{Z}_{K}^{(\mathbb{D}, \underline{\mathbb{S}})}:=K(\underline{\mathbb{D}}, \underline{\mathbb{S}})=\bigcup_{\sigma \in K} B_{\sigma}(\underline{\mathbb{D}}, \underline{\mathbb{S}}) \subset \prod_{i=1}^{m} \mathbb{D}_{i}
$$

First, for each $i \in[m]$, it follows immediately from the long exact sequence of $\left(\mathbb{D}_{i}, \mathbb{S}_{i}\right)$ that

$$
0=\widetilde{H}^{\kappa_{i}}\left(\mathbb{D}_{i} ; \mathbf{k}\right) \longrightarrow \widetilde{H}^{\kappa_{i}}\left(\mathbb{S}_{i} ; \mathbf{k}\right) \stackrel{\cong}{\longrightarrow} \widetilde{H}^{\kappa_{i}+1}\left(\mathbb{D}_{i}, \mathbb{S}_{i} ; \mathbf{k}\right) \longrightarrow \widetilde{H}^{\kappa_{i}+1}\left(\mathbb{D}_{i} ; \mathbf{k}\right)=0
$$


On the cellular cochain level, one has the following short exact sequence

$$
0 \longrightarrow C^{*}\left(\mathbb{D}_{i}, \mathbb{S}_{i} ; \mathbf{k}\right) \stackrel{j^{*}}{\longrightarrow} C^{*}\left(\mathbb{D}_{i} ; \mathbf{k}\right) \stackrel{i^{*}}{\longrightarrow} C^{*}\left(\mathbb{S}_{i} ; \mathbf{k}\right) \longrightarrow 0
$$

where each $C^{k}\left(\mathbb{D}_{i}, \mathbb{S}_{i} ; \mathbf{k}\right)$ can be considered as a subgroup of $C^{k}\left(\mathbb{D}_{i} ; \mathbf{k}\right)$, so $j^{*}$ is an inclusion. By the zig-zag lemma, one can choose a $\kappa_{i}$-cochain $x_{i}$ of $C^{\kappa_{i}}\left(\mathbb{D}_{i} ; \mathbf{k}\right)$ such that

- $i^{*}\left(x_{i}\right)$ represents a generator of $\widetilde{H}^{\kappa_{i}}\left(\mathbb{S}_{i} ; \mathbf{k}\right)$.

- $d x_{i} \in \operatorname{ker} i^{*}=\operatorname{Im} j^{*}$ so $j^{*}\left(d x_{i}\right)=d x_{i}$ may be regarded as a cocycle in $C^{\kappa_{i}+1}\left(\mathbb{D}_{i}, \mathbb{S}_{i} ; \mathbf{k}\right)$ since $j^{*}$ is an inclusion, where $d$ is the coboundary operator of $C^{*}\left(\mathbb{D}_{i} ; \mathbf{k}\right)$. Thus, the cohomological class of $d x_{i}$ generates $\widetilde{H}^{\kappa_{i}+1}\left(\mathbb{D}_{i}, \mathbb{S}_{i} ; \mathbf{k}\right)$.

Write $x_{i}^{(1)}=x_{i}$ and $x_{i}^{(2)}=d x_{i}$, and let $x_{i}^{(0)}$ denote the constant 0-cochain 1 in $C^{0}\left(\mathbb{D}_{i} ; \mathbf{k}\right)$. Obviously, $x_{i}^{(0)}, x_{i}^{(1)}$ and $x_{i}^{(2)}$ are linearly independent in $C^{*}\left(\mathbb{D}_{i} ; \mathbf{k}\right)$ as a $\mathbf{k}$-vector space.

Now let us work in the cellular cochain complex $C^{*}\left(\prod_{i=1}^{m} \mathbb{D}_{i} ; \mathbf{k}\right)$ of the product space $\prod_{i=1}^{m} \mathbb{D}_{i}$. Let $\Omega^{*}$ be the vector subspace of $C^{*}\left(\prod_{i=1}^{m} \mathbb{D}_{i} ; \mathbf{k}\right)$ spanned by the following cross products

$$
x_{1}^{\left(k_{1}\right)} \times \cdots \times x_{m}^{\left(k_{m}\right)}, \quad k_{i} \in\{0,1,2\} .
$$

An easy observation shows that $\Omega^{*}$ is a cochain subcomplex of $C^{*}\left(\prod_{i=1}^{m} \mathbb{D}_{i} ; \mathbf{k}\right)$, and $\left\{x_{1}^{\left(k_{1}\right)} \times \cdots \times x_{m}^{\left(k_{m}\right)} \mid k_{i} \in\{0,1,2\}\right\}$ forms a basis of $\Omega^{*}$ as a vector space over $\mathbf{k}$ since $x_{i}^{(0)}, x_{i}^{(1)}$ and $x_{i}^{(2)}$ are linearly independent in $C^{*}\left(\mathbb{D}_{i} ; \mathbf{k}\right)$. For convenience, we write each basis element $x_{1}^{\left(k_{1}\right)} \times \cdots \times x_{m}^{\left(k_{m}\right)}$ of $\Omega^{*}$ in the following form:

$$
\mathbf{x}^{(\tau, \sigma)}
$$

where $\mathbf{x}=x_{1}^{\left(k_{1}\right)}, \ldots, x_{m}^{\left(k_{m}\right)}, \tau=\left\{i \mid k_{i}=1\right\}$ and $\sigma=\left\{i \mid k_{i}=2\right\}$. In particular, if $\tau=$ $\sigma=\emptyset$, then $\mathbf{x}^{(\emptyset, \emptyset)}=x_{1}^{(0)} \times \cdots \times x_{m}^{(0)}$. Thus, $\Omega^{*}$ can be expressed as

$$
\Omega^{*}=\operatorname{Span}\left\{\mathbf{x}^{(\tau, \sigma)} \mid \tau, \sigma \subseteq[m] \text { with } \tau \cap \sigma=\emptyset\right\} .
$$

Next by $\Phi_{K}$ we denote the composition

$$
\Omega^{*} \hookrightarrow C^{*}\left(\prod_{i=1}^{m} \mathbb{D}_{i} ; \mathbf{k}\right) \stackrel{l^{*}}{\longrightarrow} C^{*}\left(\mathcal{Z}_{K}^{(\mathbb{D}, \underline{\mathbb{S}})} ; \mathbf{k}\right)
$$

where the latter map $l^{*}$ is induced by the inclusion $l: \mathcal{Z}_{K}^{(\mathbb{D}, \mathbb{S})} \hookrightarrow \prod_{i=1}^{m} \mathbb{D}_{i}$, and is surjective. Set

$$
S_{K}=\operatorname{Span}\left\{\mathbf{x}^{(\tau, \sigma)} \in \Omega^{*} \mid \sigma \notin K\right\} .
$$

Clearly $S_{K}$ is a cochain subcomplex of $\Omega^{*}$.

Lemma 4.1 $S_{K} \subseteq \operatorname{ker} \Phi_{K}$. Furthermore, $\Phi_{K}$ induces a cochain map $\Omega^{*} / S_{K} \longrightarrow$ $C^{*}\left(\mathcal{Z}_{K}^{(\mathbb{D}, \underline{\mathbb{S}})} ; \mathbf{k}\right)$, also denoted by $\Phi_{K}$. 
Proof Let $\mathbf{x}^{(\tau, \sigma)}$ be a basis element in $S_{K} \subset C^{*}\left(\prod_{i=1}^{m} \mathbb{D}_{i} ; \mathbf{k}\right)$. For any product cell $e=e_{1} \times \cdots \times e_{m} \subset \mathcal{Z}_{K}^{(\mathbb{D}, \mathbb{S})} \subseteq \prod_{i=1}^{m} \mathbb{D}_{i}$, there must be some $\sigma^{\prime} \in K$ such that $e \subset B_{\sigma^{\prime}}(\underline{\mathbb{D}}, \underline{\mathbb{S}})$, where each $e_{i}$ can represent a generator in the cellular chain group $C_{\operatorname{dim} e_{i}}\left(\mathbb{D}_{i} ; \mathbf{k}\right)$. In addition, it is easy to see that $e$ can also be regarded as a generator of the cellular chain complex $C_{*}\left(\mathcal{Z}_{K}^{(\mathbb{D}, \underline{\mathbb{S}})} ; \mathbf{k}\right) \stackrel{l_{*}}{\hookrightarrow} C_{*}\left(\prod_{i=1}^{m} \mathbb{D}_{i} ; \mathbf{k}\right)$ where $l_{*}$ is the inclusion induced by $l: \mathcal{Z}_{K}^{(\mathbb{D}, \underline{\mathbb{S}})} \hookrightarrow \prod_{i=1}^{m} \mathbb{D}_{i}$. Since $\sigma \notin K, \sigma$ is non-empty. Moreover, there is some $i_{0} \in \sigma \backslash \sigma^{\prime}$ such that $e_{i_{0}} \subset \mathbb{S}_{i_{0}} \subset \mathbb{D}_{i_{0}}$ and the factor $x_{i_{0}}^{(2)} \in$ $C^{\kappa_{i}+1}\left(\mathbb{D}_{i_{0}}, \mathbb{S}_{i_{0}} ; \mathbf{k}\right) \subset C^{\kappa_{i_{0}}+1}\left(\mathbb{D}_{i_{0}} ; \mathbf{k}\right)$ in $\mathbf{x}^{(\tau, \sigma)}$, together yielding that $\left\langle x_{i_{0}}^{(2)}, e_{i_{0}}\right\rangle=0$. Therefore, $\left\langle\mathbf{x}^{(\tau, \sigma)}, l_{*}(e)\right\rangle=\left\langle\mathbf{x}^{(\tau, \sigma)}, e\right\rangle=0$ by the definition of cross product. Furthermore, we see that the value of $\Phi_{K}\left(\mathbf{x}^{(\tau, \sigma)}\right)$ on $e$ is

$$
\left\langle\Phi_{K}\left(\mathbf{x}^{(\tau, \sigma)}\right), e\right\rangle=\left\langle\mathbf{x}^{(\tau, \sigma)} \circ l_{*}, e\right\rangle=\left\langle\mathbf{x}^{(\tau, \sigma)}, l_{*}(e)\right\rangle=0
$$

so $\Phi_{K}\left(\mathbf{x}^{(\tau, \sigma)}\right)=0$ in $C^{*}\left(\mathcal{Z}_{K}^{(\mathbb{D}, \underline{\mathbb{S}})} ; \mathbf{k}\right)$, as desired.

By $\Omega^{*}(K)$ we denote the quotient $\Omega^{*} / S_{K}$. Let $L$ be a subcomplex of $K$. Then we obtain a pair $\left(\mathcal{Z}_{K}^{(\mathbb{D}, \underline{\mathbb{S}})}, \mathcal{Z}_{L}^{(\mathbb{D}, \underline{\mathbb{S}})}\right)$ of $\mathrm{CW}$-complexes. Now since $S_{K} \subseteq S_{L}$, we have a short exact sequence

$$
0 \longrightarrow \operatorname{ker} \pi^{*} \longrightarrow \Omega^{*}(K) \stackrel{\pi^{*}}{\longrightarrow} \Omega^{*}(L) \longrightarrow 0
$$

where $\pi^{*}$ is induced by the natural inclusion $\pi: S_{K} \hookrightarrow S_{L}$. By $\Omega^{*}(K, L)$ we denote the kernel ker $\pi^{*}$. It is easy to see that two cochain maps $\Phi_{K}: \Omega^{*}(K) \longrightarrow$ $C^{*}\left(\mathcal{Z}_{K}^{(\mathbb{D}, \underline{\mathbb{S}})} ; \mathbf{k}\right)$ and $\Phi_{L}: \Omega^{*}(L) \longrightarrow C^{*}\left(\mathcal{Z}_{L}^{(\mathbb{D}, \underline{\mathbb{S}})} ; \mathbf{k}\right)$ give a cochain map $\Phi_{(K, L)}$ : $\Omega^{*}(K, L) \longrightarrow C^{*}\left(\mathcal{Z}_{K}^{(\mathbb{D}, \underline{\mathbb{S}})}, \mathcal{Z}_{L}^{(\mathbb{D}, \underline{\mathbb{S}})} ; \mathbf{k}\right)$ such that the following diagram commutes

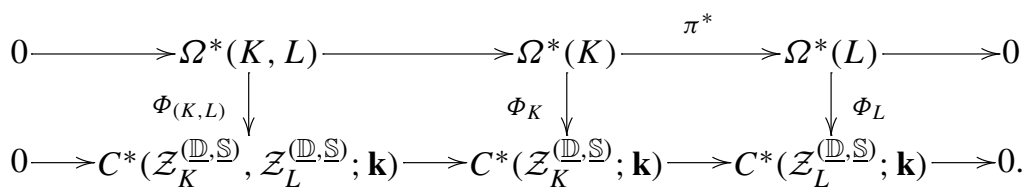

Furthermore, we may obtain a homomorphism between two long exact cohomology sequences given by two short exact sequences above.

Proposition 4.1 For any $K \in \mathcal{K}_{[m]}, \Phi_{K}$ induces an isomorphism

$$
H^{*}\left(\Omega^{*}(K) ; \mathbf{k}\right) \stackrel{\cong}{\longrightarrow} H^{*}\left(\mathcal{Z}_{K}^{(\mathbb{D}, \underline{\mathbb{S}})} ; \mathbf{k}\right)
$$

as graded $\mathbf{k}$-modules.

Proof First observe that for $K=\{\emptyset\}, \Omega^{*}(K)$ is spanned by $\left\{\mathbf{x}^{(\tau, \emptyset)} \mid \tau \subseteq[m]\right\}$ with zero coboundary operator. On the other hand, if $K=\{\emptyset\}$ then $\mathcal{Z}_{K}^{(\mathbb{D}, \mathbb{S})}=\prod_{i=1}^{m} \mathbb{S}_{i}$. By the Künneth formula, the above set is not only a set but also a basis of $H^{*}\left(\mathcal{Z}_{K}^{(\mathbb{D}, \underline{\mathbb{S}})} ; \mathbf{k}\right)$ 
as a graded $\mathbf{k}$-module (if we view the elements of the set as cohomological classes). Thus, clearly $\Phi_{K}$ induces an isomorphism in this case.

Next we proceed inductively by considering a pair of abstract simplicial complexes $(K, L)$ where $K=L \sqcup\left\{\sigma_{0}\right\}$ for some simplex $\sigma_{0}$ (which is a maximal element of $K$ as a poset). Hence $\left(\mathcal{Z}_{K}^{(\mathbb{D}, \mathbb{S})}, \mathcal{Z}_{L}^{(\mathbb{D}, \underline{\mathbb{S}})}\right)$ is a pair of $C W$-complexes, which has by excision the same cohomology as $\left(\mathcal{Z}_{2^{\sigma_{0}}}^{(\mathbb{D}, \underline{\mathbb{S}})}, \mathcal{Z}_{2^{\sigma}}^{\left(\frac{\mathbb{D}}{\mathbb{S}} \backslash\left\{\sigma_{0}\right\}\right.}\right)$. This pair $\left(\mathcal{Z}_{2^{\sigma_{0}}}^{(\mathbb{D}, \underline{\mathbb{S}})}, \mathcal{Z}_{2^{\sigma_{0}} \backslash\left\{\sigma_{0}\right\}}^{(\mathbb{\mathbb { S }})}\right)$ is in turn homeomorphic to

$$
\prod_{i \in[m] \backslash \sigma_{0}} \mathbb{S}_{i} \times\left(\prod_{i \in \sigma_{0}} \mathbb{D}_{i}, A\left(\prod_{i \in \sigma_{0}} \mathbb{D}_{i}\right)\right)
$$

where $A\left(\prod_{i \in \sigma_{0}} \mathbb{D}_{i}\right)=\left(\mathbb{S}_{i_{1}} \times \mathbb{D}_{i_{2}} \times \cdots \times \mathbb{D}_{i_{s}}\right) \cup \cdots \cup\left(\mathbb{D}_{i_{1}} \times \cdots \times \mathbb{D}_{i_{s-1}} \times \mathbb{S}_{i_{s}}\right)$ with $\sigma_{0}=\left\{i_{1}, \ldots, i_{s} \mid i_{1}<\cdots<i_{s}\right\}$. By relative Künneth formula, its cohomology with $\mathbf{k}$ coefficients is isomorphic to

$$
\operatorname{Span}\left\{\mathbf{x}^{\left(\tau, \sigma_{0}\right)} \mid \tau \subseteq[m] \text { with } \tau \cap \sigma_{0}=\emptyset\right\}
$$

as graded k-modules. On the other hand, we see easily from the short exact sequence (4.1) that $\Omega^{*}(K, L)=\operatorname{ker} \pi^{*}$ is exactly equal to the cochain complex

$$
\operatorname{Span}\left\{\mathbf{x}^{\left(\tau, \sigma_{0}\right)} \mid \tau \subseteq[m] \text { with } \tau \cap \sigma_{0}=\emptyset\right\}
$$

with zero coboundary operator. It then follows that $\Phi_{(K, L)}$ induces an isomorphism

$$
H^{*}\left(\Omega^{*}(K, L) ; \mathbf{k}\right) \stackrel{\cong}{\longrightarrow} H^{*}\left(\mathcal{Z}_{K}^{(\mathbb{D}, \underline{\mathbb{S}})}, \mathcal{Z}_{L}^{(\mathbb{D}, \underline{\mathbb{S}})} ; \mathbf{k}\right)
$$

as graded k-modules. Inductively, now we may assume that $\Phi_{L}$ induces an isomorphism $H^{*}\left(\Omega^{*}(L) ; \mathbf{k}\right) \longrightarrow H^{*}\left(\mathcal{Z}_{L}^{(\mathbb{D}, \mathbb{S})} ; \mathbf{k}\right)$ as graded $\mathbf{k}$-modules. Hence we may conclude that the same holds for $H^{*}\left(\Omega^{*}(K) ; \mathbf{k}\right) \longrightarrow H^{*}\left(\mathcal{Z}_{K}^{(\mathbb{D}, \mathbb{S})} ; \mathbf{k}\right)$ by the five-lemma. This completes the induction and the proof of Proposition 4.1.

Now let us return to study the complex $\left(\Omega^{*}(K), \underline{d}\right)$. First, we may impose a $\{0,1\}^{m}$-graded (or $2^{[m]}$-graded) structure on $\Omega^{*}(K)$, by defining for $a \in 2^{[m]}$

$$
\Omega^{*}(K)_{a}:=\operatorname{Span}\left\{\mathbf{x}^{(\tau, \sigma)} \mid \tau \subseteq[m], \sigma \in K \text { with } \tau \cup \sigma=a, \tau \cap \sigma=\emptyset\right\} .
$$

Then, clearly $\Omega^{*}(K)=\bigoplus_{a \in 2^{[m]}} \Omega^{*}(K)_{a}$. Furthermore, given a basis element $\mathbf{x}^{(\tau, \sigma)} \in \Omega^{*}(K)_{a}$ with $\tau=a \backslash \sigma$, by a direct calculation we have

$$
\underline{d}\left(\mathbf{x}^{(a \backslash \sigma, \sigma)}\right)=\sum_{\substack{k \in a \backslash \sigma \\ \sigma \cup\{k\} \in K}} \epsilon_{k} \mathbf{x}^{(a \backslash(\sigma \cup\{k\}), \sigma \cup\{k\})}
$$

which still belongs to $\Omega^{*}(K)_{a}$, where $\epsilon_{k}= \pm 1$. So $\left(\Omega^{*}(K)_{a}, \underline{d}\right)$ has also a cochain complex structure. This means that $\Omega^{*}(K)$ is a bigraded k-module. Also, clearly the basis of $\Omega^{*}(K)_{a}$ is indexed by $\left.K\right|_{a}$ where $\left.K\right|_{a}=\{\sigma \in K \mid \sigma \subseteq a\}$. 
Lemma 4.2 For each $a \in 2^{[m]},\left(\Omega^{*}(K)_{a}, \underline{d}\right)$ is isomorphic to the coaugmented cochain complex $\left(C^{*}\left(\left.K\right|_{a} ; \mathbf{k}\right), d^{\prime}\right)$ as cochain complexes. Furthermore, $H^{*}\left(\Omega^{*}(K)_{a} ; \mathbf{k}\right) \cong \widetilde{H}^{*}\left(\left.K\right|_{a} ; \mathbf{k}\right)$ as graded $\mathbf{k}$-modules.

Lemma 4.2 is a (dualized) consequence of the following general result.

Lemma 4.3 Let $K$ be an abstract simplicial complex on a finite set. Let $V(K)$ be a vector space over $\mathbf{k}$ with a $K$-indexed basis $\left\{v_{\sigma} \mid \sigma \in K\right\}$, and let $\iota: V(K) \longrightarrow V(K)$ be a linear map such that $\iota^{2}=0$ and $\iota\left(v_{\sigma}\right)=\sum_{k \in \sigma} \varepsilon_{k} v_{\sigma \backslash\{k\}}$ where $\varepsilon_{k}= \pm 1$. Then there is an isomorphism $f: V(K) \longrightarrow C_{*}(K ; \mathbf{k})$ as $\mathbf{k}$-vector spaces with form $f$ : $v_{\sigma} \longmapsto \varepsilon_{\sigma} \sigma$ such that $f \circ \iota=\partial \circ f$, where $\varepsilon_{\sigma}= \pm 1$ and $C_{*}(K ; \mathbf{k})$ is the ordinary chain complex over $\mathbf{k}$ of $K$ with the boundary operator $\partial$.

Proof We proceed inductively. For $K=\{\emptyset\}, V(K)=\operatorname{Span}\left\{v_{\emptyset}\right\} \cong \mathbf{k}$ with $\iota=0$ and $C_{*}(K ; \mathbf{k})=\operatorname{Span}\{\emptyset\} \cong \mathbf{k}$ with $\partial=0$, so clearly we have such an $f$. Now for an arbitrary $K \neq\{\emptyset\}$, take a maximal element $\sigma_{0}$ of $K$ (as a poset) so that $L=K \backslash\left\{\sigma_{0}\right\}$ is a subcomplex of $K$. The subspace $\left.V(K)\right|_{L}=\operatorname{Span}\left\{v_{\sigma} \mid \sigma \in L\right\}$ is invariant under $\iota$. So we can apply induction hypothesis to $\left(\left.V(K)\right|_{L}, \iota\right)$, yielding an isomorphism $f_{0}$ : $\left.V(K)\right|_{L} \longrightarrow C_{*}(L ; \mathbf{k})$ by $v_{\sigma} \longmapsto \varepsilon_{\sigma} \sigma$ such that $f_{0} \circ \iota=\partial \circ f_{0}$. Now observe that $\iota\left(v_{\sigma_{0}}\right)=\left.\sum_{k \in \sigma_{0}} \varepsilon_{k} v_{\sigma_{0} \backslash\{k\}} \in V(K)\right|_{L}$, so $f_{0}\left(\iota\left(v_{\sigma_{0}}\right)\right)=\sum_{k \in \sigma_{0}} \varepsilon_{k} \varepsilon_{\sigma_{0} \backslash\{k\}}\left(\sigma_{0} \backslash\{k\}\right)$ which is in the chain group $C_{\left|\sigma_{0}\right|-2}\left(2^{\sigma_{0}} ; \mathbf{k}\right) \subset C_{\left|\sigma_{0}\right|-2}(L ; \mathbf{k})$, and $\left(\partial \circ f_{0}\right)\left(\iota\left(v_{\sigma_{0}}\right)\right)=\left(f_{0} \circ\right.$ $\iota)\left(\iota\left(v_{\sigma_{0}}\right)\right)=f_{0}\left(\iota^{2}\left(v_{\sigma_{0}}\right)\right)=0$, i.e., $f_{0}\left(\iota\left(v_{\sigma_{0}}\right)\right) \in \operatorname{ker} \partial$. Since $C_{*}\left(2^{\sigma_{0}} ; \mathbf{k}\right)$ is acyclic and $C_{\left|\sigma_{0}\right|-1}\left(2^{\sigma_{0}} ; \mathbf{k}\right)=\operatorname{Span}\left\{\sigma_{0}\right\}$, we have $f_{0}\left(\iota\left(v_{\sigma_{0}}\right)\right)=\partial\left(n \sigma_{0}\right)$ for some $n \in \mathbf{k}$. However, $\partial\left(n \sigma_{0}\right)=n \partial\left(\sigma_{0}\right)$ so $n \partial\left(\sigma_{0}\right)=\sum_{k \in \sigma_{0}} \varepsilon_{k} \varepsilon_{\sigma_{0} \backslash\{k\}}\left(\sigma_{0} \backslash\{k\}\right)$. This forces $n$ to be \pm 1 . We can then extend $f_{0}$ to $f: V(K) \longrightarrow C_{*}\left(K\right.$; k) by defining $v_{\sigma_{0}} \longmapsto n \sigma_{0}$, so that we have

$$
f\left(\iota\left(v_{\sigma_{0}}\right)\right)=f_{0}\left(\iota\left(v_{\sigma_{0}}\right)\right)=\partial\left(n \sigma_{0}\right)=\partial\left(f\left(v_{\sigma_{0}}\right)\right) .
$$

Hence $f \circ \iota=\partial \circ f$ in $V(K)$. The induction step is finished, proving the lemma. $2^{[m]}$,

The famous Hochster formula tells us (see [14, Corollary 5.12]) that for each $a \in$

$$
\widetilde{H}^{|a|-i-1}\left(\left.K\right|_{a} ; \mathbf{k}\right) \cong \operatorname{Tor}_{i}^{\mathbf{k}[\mathbf{v}]}(\mathbf{k}(K), \mathbf{k})_{a} .
$$

We know by Lemma 4.2 that each class of $\widetilde{H}^{|a|-i-1}\left(\left.K\right|_{a} ; \mathbf{k}\right)$ may be understood as one of $H^{*}\left(\Omega^{*}(K)_{a} ; \mathbf{k}\right)$, represented by a linear combination of the elements of the form $\mathbf{x}^{(a \backslash \sigma, \sigma)} \in \Omega^{*}(K)_{a}$ with $|\sigma|=|a|-i$; so by Proposition 4.1 it corresponds to a cohomological class of degree $|\sigma|+\sum_{k \in a} \kappa_{k}=-i+\sum_{k \in a}\left(\kappa_{k}+1\right)$ in $H^{*}\left(\mathcal{Z}_{K}^{(\mathbb{D}, \mathbb{S})} ; \mathbf{k}\right)$. To sum up, it follows that for each $n \geq 0$,

$$
H^{n}\left(\mathcal{Z}_{K}^{(\mathbb{D}, \underline{\mathbb{S}})} ; \mathbf{k}\right) \cong \bigoplus_{\substack{a \in 2^{[m]} \\-i+\sum_{k \in a}\left(\kappa_{k}+1\right)=n}} \operatorname{Tor}_{i}^{\mathbf{k}[\mathbf{v}]}(\mathbf{k}(K), \mathbf{k})_{a}
$$

Combining with all arguments above, we conclude that 
Theorem 4.2 As graded $\mathbf{k}$-modules,

$$
H^{*}\left(\mathcal{Z}_{K}^{(\mathbb{D}, \underline{\mathbb{S}})} ; \mathbf{k}\right) \cong \operatorname{Tor}^{\mathbf{k}[\mathbf{v}]}(\mathbf{k}(K), \mathbf{k})
$$

Together with Proposition 2.2 and Theorem 4.2, we obtain that

$$
\text { Corollary 4.3 } \sum_{i} \operatorname{dim}_{\mathbf{k}} H^{i}\left(\mathcal{Z}_{K}^{(\mathbb{D}, \underline{\mathbb{S}})} ; \mathbf{k}\right)=\sum_{i=0}^{h} \sum_{a \in 2^{[m]}} \beta_{i, a}^{\mathbf{k}(K)} \text {. }
$$

Remark 9 It should be pointed out that here we merely determine the $\mathbf{k}$-module structure of $H^{*}\left(\mathcal{Z}_{K}^{(\mathbb{D}, \mathbb{S})} ; \mathbf{k}\right)$. Of course, this is enough for our purpose in this paper. Observe that if there are two $i, j \in[m]$ with $i \neq j$ such that $\kappa_{i}$ and $\kappa_{j}$ are even, then for $x_{i}^{(2)}, x_{j}^{(2)} \in \Omega^{*}(K), x_{i}^{(2)} \times x_{j}^{(2)}=-x_{j}^{(2)} \times x_{i}^{(2)}$. This means that in this case, if $\mathbf{k}$ is not a field of characteristic 2 , then $H^{*}\left(\Omega^{*}(K)\right.$; $\left.\mathbf{k}\right)$ cannot be isomorphic to $\operatorname{Tor}^{\mathbf{k}[\mathbf{v}]}(\mathbf{k}(K), \mathbf{k})$ as $\mathbf{k}$-algebras since $\mathbf{k}(K)$ is a commutative ring. Even when $\mathbf{k}$ is a field of characteristic 2 , there is still some nuance preventing us from simply extending the ring structure result (4.1) of Buchstaber and Panov to the case of, say $\mathbb{R} \mathcal{Z}_{K}$; Indeed, in this case $x_{i}^{(1)}$ would be a 0-cochain, which satisfies $x_{i}^{(1)} \cup x_{i}^{(1)}=x_{i}^{(1)}$, whereas in the cases when $\kappa_{i}>0, x_{i}^{(1)} \cup x_{i}^{(1)}$ would be instead zero element in $H^{*}\left(\mathbb{S}_{i} ; \mathbf{k}\right)$. Nevertheless, our calculation of the module structure actually represents any cohomological class in $H^{*}\left(\mathcal{Z}_{K}^{(\mathbb{D}, \mathbb{S})} ; \mathbf{k}\right)$ as a sum of $\mathbf{x}^{(\tau, \sigma)}$ 's via the isomorphism $H^{*}\left(\Omega^{*}(K) ; \mathbf{k}\right) \cong H^{*}\left(\mathcal{Z}_{K}^{(\mathbb{D}, \underline{\mathbb{S}})} ; \mathbf{k}\right)$, from which we may also figure out the cohomological equivalence relation amongst such sums; since the cup product of pairs of these elements is clear, in a certain sense we should have also determined the ring structure of $H^{*}\left(\mathcal{Z}_{K}^{(\mathbb{D}, \underline{\mathbb{S}})} ; \mathbf{k}\right)$. In other words, let $\mathbf{k}(K)=\mathbf{k}[\mathbf{v}] / I_{K}=\mathbf{k}\left[v_{1}, \ldots, v_{m}\right] / I_{K}$ be the Stanley-Reisner face ring of $K$ with $\operatorname{deg} v_{i}=\kappa_{i}+1$. Then it should be reasonable to conjecture that the following results hold:

- If all $\kappa_{i}$ 's are odd, then $H^{*}\left(\mathcal{Z}_{K}^{(\mathbb{D}, \underline{\mathbb{S}})} ; \mathbf{k}\right) \cong \operatorname{Tor}^{\mathbf{k}[\mathbf{v}]}(\mathbf{k}(K), \mathbf{k})$ as $\mathbf{k}$-algebras.

- If $\kappa_{i}>0$ for any $i \in[m]$, then $H^{*}\left(\mathcal{Z}_{K}^{(\mathbb{D}, \underline{\mathbb{S}})} ; \mathbf{k}_{2}\right) \cong \operatorname{Tor}^{\mathbf{k}_{2}[\mathbf{v}]}\left(\mathbf{k}_{2}(K), \mathbf{k}_{2}\right)$ as $\mathbf{k}_{2}$ algebras.

- In general, $H^{*}\left(\mathcal{Z}_{K}^{(\mathbb{D}, \underline{\mathbb{S}})} ; \mathbf{k}_{2}\right) \cong H\left[H^{*}\left(\prod_{i=1}^{m} \mathbb{S}_{i} ; \mathbf{k}_{2}\right) \otimes_{\mathbf{k}_{2}[\mathbf{v}]} \mathbf{k}_{2}(K)\right]$ as $\mathbf{k}_{2}$-algebras.

\section{Application to the free actions on $\mathcal{Z}_{K}$ and $\mathbb{R} \mathcal{Z}_{K}$}

First we prove a useful lemma.

Lemma 5.1 Let $K \in \mathcal{K}_{[m]}$ be an abstract simplicial complex on vertex set $[m]$, and let $H$ (resp. $\left.H_{\mathbb{R}}\right)$ be a rank $r$ subtorus of $T^{m}$ (resp. $\left.\left(\mathbb{Z}_{2}\right)^{m}\right)$. If the restricted action of $\Phi$ to $H\left(\right.$ resp. $\Phi_{\mathbb{R}}$ to $\left.H_{\mathbb{R}}\right)$ is free on $\mathcal{Z}_{K}\left(\right.$ resp. $\left.\mathbb{R} \mathcal{Z}_{K}\right)$, then $r \leq m-\operatorname{dim} K-1$.

Proof It is well-known that the restricted action of $\Phi$ to $H$ (resp. $\Phi_{\mathbb{R}}$ to $H_{\mathbb{R}}$ ) is free on $\mathcal{Z}_{K}$ (resp. $\mathbb{R} \mathcal{Z}_{K}$ ) if and only if for any point $z$ (resp. $x$ ) of $\mathcal{Z}_{K}$ (resp. $\mathbb{R} \mathcal{Z}_{K}$ ), $H \cap G_{z}$ (resp. $H_{\mathbb{R}} \cap G_{x}$ ) is trivial, where $G_{z}$ (resp. $G_{x}$ ) is the isotropy subgroup at $z$ (resp. $x$ ) of the $T^{m}$-action $\Phi$ (resp. the $\left(\mathbb{Z}_{2}\right)^{m}$-action $\left.\Phi_{\mathbb{R}}\right)$. Suppose that $r>$ 
$m-\operatorname{dim} K-1$. Take $a \in K$ with $|a|=\operatorname{dim} K+1$. Without the loss of generality, assume that $a=\{1, \ldots,|a|\}$. Then we see that $\mathcal{Z}_{K}$ (resp. $\mathbb{R} \mathcal{Z}_{K}$ ) contains the point of the form $z=\left(0, \ldots, 0, z_{|a|+1}, \ldots, z_{m}\right)$ (resp. $\left.x=\left(0, \ldots, 0, x_{|a|+1}, \ldots, x_{m}\right)\right)$. It is easy to see that the isotropy subgroup $G_{z}$ (resp. $G_{x}$ ) has rank at least $|a|$, so the intersection $H \cap G_{z}$ (resp. $H_{\mathbb{R}} \cap G_{x}$ ) cannot be trivial. This contradiction means that $r$ must be equal to or less than $m-\operatorname{dim} K-1$.

Now let us use the preceding results to complete the proof of Theorem 1.4.

Proof Theorem 1.4 Let $f=\sum_{a \in K} \delta_{a} \in \mathcal{F}_{[m]}$ such that $\operatorname{supp}(f)=K$. If $f=\underline{1}$ (i.e., $\left.K=2^{[m]}\right)$, then $\mathcal{Z}_{K}=\left(D^{2}\right)^{m}$ (resp. $\left.\mathbb{R} \mathcal{Z}_{K}=\left(D^{1}\right)^{m}\right)$. However, any properly nontrivial subtorus of $T^{m}$ (resp. $\left.\left(\mathbb{Z}_{2}\right)^{m}\right)$ cannot freely act on $\left(D^{2}\right)^{m}$ (resp. $\left.\left(D^{1}\right)^{m}\right)$ since the point $(0, \ldots, 0)$ is always a fixed point. Thus we may assume that $f \neq 1$. By Theorem 3.5, there exists some $a \in 2^{[m]}$ with $a \neq[m]$ such that $a \in \operatorname{supp}(f)=K$ and $|\operatorname{supp}(\mathcal{M}(f))| \geq 2^{m-n}$ where $n=|a|$. Since $a \in K$, we have $n \leq \operatorname{dim} K+1$. So by Lemma 5.1 it follows that $n \leq m-r$ and $r \leq m-n$. Combining with Theorem 3.5 and Corollaries 3.2 and 4.3 together gives

$$
2^{r} \leq 2^{m-n} \leq|\operatorname{supp}(\mathcal{M}(f))| \leq \sum_{i} \operatorname{dim}_{\mathbf{k}} H^{i}\left(\mathcal{Z}_{K} ; \mathbf{k}\right)=\sum_{i} \operatorname{dim}_{\mathbf{k}} H^{i}\left(\mathbb{R} \mathcal{Z}_{K} ; \mathbf{k}\right)
$$

as desired.

Acknowledgements The authors are grateful to M. Franz, M. Masuda, T.E. Panov, V. Puppe and L. Yu for their comments and suggestions. In particular, the authors also express their gratitude to the referees, who did an extremely careful reading of this paper and gave many valuable suggestions and comments.

\section{References}

1. Adem, A., Browder, W.: The free rank of symmetry of $\left(S^{n}\right)^{k}$. Invent. Math. 92, 431-440 (1988)

2. Adem, A., Davis, J.F.: Topics in transformation groups. In: Handbook of Geometric Topology, pp. 154. North-Holland, Amsterdam (2002)

3. Allday, C., Puppe, V.: Cohomological Methods in Transformation Groups. Cambridge Studies in Advanced Mathematics, vol. 32. Cambridge University Press, Cambridge (1993)

4. Bahri, A., Bendersky, M., Cohen, F.R., Gitler, S.: Decompositions of the polyhedral product functor with applications to moment-angle complexes and related spaces. Proc. Natl. Acad. Sci. USA 106, 12241-12244 (2009)

5. Buchstaber, V.M., Panov, T.E.: Torus Actions and Their Applications in Topology and Combinatorics. University Lecture Series, vol. 24. Amer. Math. Soc., Providence (2002)

6. Buchstaber, V.M., Panov, T.E.: Combinatorics of simplicial cell complexes and torus action. Proc. Steklov Inst. Math. 247, 33-49 (2004)

7. Carlsson, G.: On the non-existence of free actions of elementary abelian groups on products of spheres. Am. J. Math. 102, 1147-1157 (1980)

8. Carlsson, G.: On the rank of abelian groups acting freely on $\left(S^{n}\right)^{k}$. Invent. Math. 69, 393-400 (1982)

9. Carlsson, G.: Free $(\mathbb{Z} / 2)^{k}$-actions and a problem in commutative algebra. In: Transformation Groups, Poznań 1985, Lecture Notes in Math., vol. 1217, pp. 79-83. Springer, Berlin (1986)

10. Conner, P.E.: On the action of a finite group on $S^{n} \times S^{n}$. Ann. Math. 66, 586-588 (1957)

11. Davis, M.W., Januszkiewicz, T.: Convex polytopes, Coxeter orbifolds and torus actions. Duke Math. J. 62, 417-451 (1991)

12. Denham, G., Suciu, A.: Moment-angle complexes, monomial ideals and Massey products. Pure Appl. Math. Q. 3, 25-60 (2007) 
13. Halperin, S.: Rational homotopy and torus actions. In: Aspects of Topology, London Math. Soc. Lecture Note Ser., vol. 93, pp. 293-306. Cambridge University Press, Cambridge (1985)

14. Miller, E., Sturmfels, B.: Combinatorial Commutative Algebra. Graduate Texts in Math., vol. 227. Springer, Berlin (2005)

15. Panov, T.E.: Cohomology of face rings and torus actions. Lond. Math. Soc. Lect. Note Ser. 347, 165-201 (2008)

16. Puppe, V.: Multiplicative aspects of the Halperin-Carlsson conjecture. Georgian Math. J. 16, 369-379 (2009)

17. Smith, P.A.: Orbit spaces of abelian p-groups. Proc. Natl. Acad. Sci. USA 45, 1772-1775 (1959)

18. Yalcin, E.: Groups actions and group extensions. Trans. Am. Math. Soc. 352, 2689-2700 (2000) 